\title{
Practical identifiability of electrochemical P2D models for lithium-ion batteries
}

\author{
Vincent Laue ${ }^{1} \cdot$ Fridolin Röder $^{1} \cdot$ Ulrike Krewer $^{1,2}$ (1)
}

Received: 20 November 2020 / Accepted: 15 May 2021 / Published online: 26 June 2021

(c) The Author(s) 2021

\begin{abstract}
Electrochemical models play a significant role in today's rapid development and enhancement of lithium-ion batteries. For instance, they are applied for design and process optimization. More recently, model and parameter identifiability are gaining interest as thorough model parameterization is key to reliable simulation results. Especially electrochemical models are often prone to unidentifiability and overfitting due to their high number of adjustable parameters. In this article, the most common electrochemical peudo-2D model of a lithium-ion battery is parameterized. A three-step procedure is applied which considers quasi-static 3-electrode measurements of the open-circuit potential, C-rate tests, and electrochemical impedance spectra. Identifiability of each step is discussed in-depth and a general guidance for future parameterizations is derived. The conducted study reveals the insufficiency of open-circuit potential and C-rate tests to fully parameterize the electrochemical model. Highly dynamic tests, e.g., impedance spectroscopy, are required to resolve the ambiguity of diffusive and electric processes under quasi-static conditions. Any parameterization of electrochemical models requires experimental data of electrode-resolved tests, as well as a combination of quasi-static and highly dynamic tests. The results of this study provide guidance for the use of electrochemical models in applied sciences and industry.
\end{abstract}

\section{Graphic abstract}
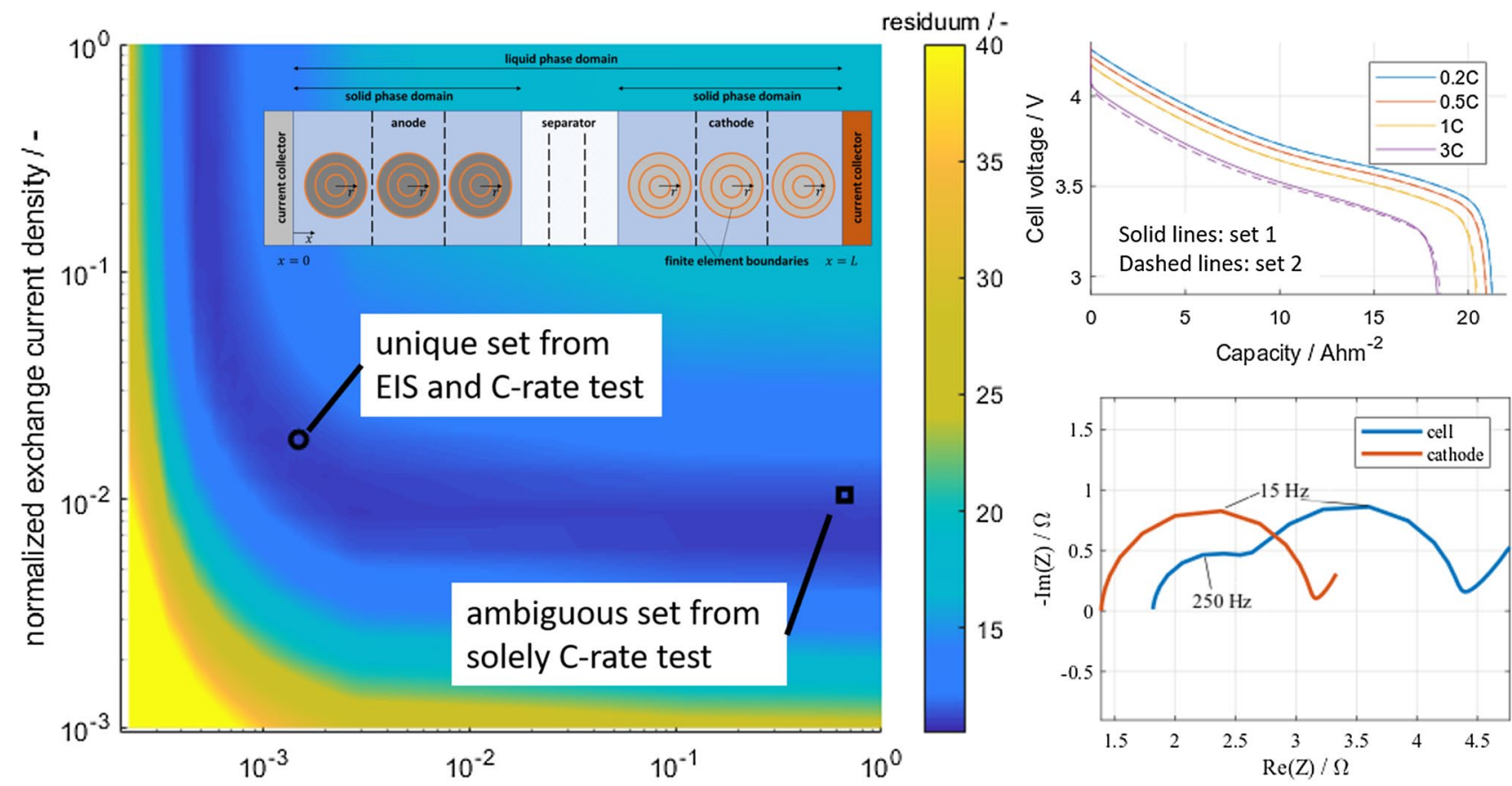

normalized conductivity and diffusivity of cathode / -

Extended author information available on the last page of the article 
Keywords Lithium-ion battery $\cdot$ Electrochemical modeling $\cdot$ Model identifiability

$\begin{array}{ll}\text { Abbreviations } \\ \text { CC } & \text { Constant current } \\ \text { CV } & \text { Constant voltage } \\ \text { DL } & \text { Double layer } \\ \text { ECM } & \text { Equivalent circuit model } \\ \text { EIS } & \text { Electrochemical impedance spectroscopy } \\ \text { FOM } & \text { Fractional-order models } \\ \text { LIB } & \text { Lithium-ion battery } \\ \text { OCP } & \text { Open-circuit potential } \\ \text { P2D } & \text { Pseudo two dimensional } \\ \text { PE } & \text { Parameter estimation } \\ \text { SEI } & \text { Solid-electrolyte interface } \\ \text { SOC } & \text { State of charge } \\ \text { SPM } & \text { Single-particle model }\end{array}$

\section{Introduction}

Nowadays, the lithium-ion battery (LIB) is the dominant electrochemical storage technology for a large variety of applications. The further development of LIBs is based on enhancements of battery materials and the extended application of novel modeling approaches used for optimization.

Due to new applications for models and a significant increase of computational power, a wide variety of models has been developed and different applications have been addressed, e.g., models have been used to quantify solid-electrolyte interface (SEI) formation and aging [14, $34,39]$. The variety of models is summarized in reviews about modeling of LIBs with focus on systems engineering, multi-scale modeling, and state estimation in electric cars, respectively [12, 19, 28, 33].
Besides the governing equations, model parameters are an essential part of every model. Their correct choice can be not only crucial but also challenging. While parameter estimation (PE) is commonly used to parameterize models, it is also a powerful tool to derive non-measurable parameters from cell performance. These yield insight, e.g., into the impact of production parameters [22]. Both applications require practical identifiability of the model parameters. In the following, parameter estimation approaches are briefly reviewed, and direct and sample-based approaches of proofing identifiability are summarized.

The most common parameters in electrochemical LIB models are reaction rate constants, diffusion coefficients of active materials and electrolyte, and conductivity of solid and electrolyte. Temperature dependencies are commonly modeled using Arrhenius' law. It has a pre-exponential coefficient and an activation energy which are estimated from temperature variations. Concentration dependencies can also be considered and may further increase the number of parameters. For instance, in Ref. [11], the state of charge (SOC) or concentration-dependent open-circuit potential (OCP) between both electrodes was estimated from electrochemical experiments alongside the other model parameters. Fitting empirical OCP equations for both electrodes together with kinetic constants. etc., led to a large number of parameters. To deconvolute the joint parameter estimation of OCP curves and other model parameters Refs. [20] and [22] first parameterized the thermodynamic, OCP-related parameters. Then, in the subsequent main parameter estimation kinetic constants, diffusion coefficients, etc., were estimated. For equivalent circuit models, resistances, capacitance, and OCP have to be estimated, and concentration dependencies are common [19].
Table 1 Number of estimated parameters in different publications in order of publication

\begin{tabular}{lllll}
\hline $\begin{array}{l}\text { Number of param- } \\
\text { eters }\end{array}$ & Year & Refs. & Model type & Experiment \\
\hline 88 & 2012 & {$[11]$} & SPM incl. OCP & Drive cycles \\
11 & 2013 & {$[26]$} & reduced-order SPM & C-rate and pulse tests \\
18 & 2014 & {$[43]$} & P2D incl. $T$ dependency & C-rate tests \\
4 & 2015 & {$[27]$} & P2D & One charge/discharge \\
3 & 2017 & {$[22]$} & P2D incl. DL & C-rate tests \\
27 & 2018 & {$[17]$} & P2D & C-rate tests \\
6 & 2018 & {$[7]$} & ECM & C-rate tests \\
14 & 2018 & {$[4]$} & P2D & CC/CV charge/discharge \\
7 & 2019 & {$[20]$} & P2D incl. DL & C-rate tests \\
\hline
\end{tabular}

Used abbreviations are double layer (DL), single-particle model (SPM), pseudo-2D model (P2D), equivalent circuit model (ECM), constant voltage (CV), and constant current (CC) 
The number of estimated parameters varies significantly between different publications. This is exemplary illustrated in Table 1 . The difference in numbers is partially related to different model complexities and types, and partially to the application of different parameter estimation procedures. Further, the number of uncertain parameters is dependent on the model application, e.g., whether temperatures are varied in the experiments and its influence is modeled.

As Table 1 shows, the most common case is a P2D model without temperature dependency in combination with C-rate tests. This commonly leads to three to 14 parameters. The difference in total number of parameters is partially related to differences in parameterization approaches and research objectives. But this differences also raises the equation about the maximum number of independent uncertain parameters which can be estimated in one model.

Different parameter procedures were introduced in the literature. For physics-based full-order models (P2D models and SPMs in Table 1), Ramadesigan et al. applied gradientbased, thus local, least-squares fitting to estimate parameter changes due to cell aging in combination with an uncertainty quantification to calculate the confidence intervals of the estimated parameters [32]. Vazquez-Arenas et al. coupled the parameter estimation with a sensitivity analysis to assess the estimation accuracy [43]. Lenze et al. estimated parameters of an electrochemical model manually adjusting parameters [22]. Chun and Han introduced a cascaded improved harmony search to estimate the parameters of the P2D model, wherein a partially random-based optimization approach was conducted repeatedly [4].

In the engineering and control community, there exists a variety of reduced-order models or equivalent circuit models (ECMs) [1, 18, 29, 45]. For ECMs, parameter estimation is frequently conducted [1]. These models commonly focus on estimation of battery states like SOC, state of power, or state of health [16, 25, 29, 42, 44]. Dvorak et al. introduced a parameter estimation method for an ECM containing 2 $\mathrm{RC}$ elements. This method applies step-wise discharge at constant current with repetitions at different C-rates and temperatures [7]. A Kalman filter was applied to the ECM. It was shown that in this case, parameter estimation should facilitate electrochemical tests similar to the actual application for optimal accordance of the model [2].

Besides the choice of the experimental-electrical test procedure and optimization algorithms, in some publications, the objective function was adjusted to enhance identifiability. Different objective functions were applied to different parts of the experimental data. For instance, Li et al. applied three steps: high SOC and low current, low SOC and low current, and eventually considered a dynamic driving cycle [23]. Jin et al. considered thermodynamic parameters and kinetic parameters independently for a P2D model as well as linear or logarithmic scaling for different parameters [17].
All these publications have a strong theoretical focus. In contrast, Ecker et al. used a variety of different experimental analysis methods to measure model parameters like diffusion coefficients. They used electrochemical impedance spectroscopy in combination with equivalent circuit models to partially estimate the parameters of the P2D model [8, 9]. Schmalstieg et al. presented a method to parameterize a model of bought-in commercial cells. Their method combined EIS, galvanostatic intermittent titration technique, porosity measurement by mercury intrusion, and ECM fitting [36].

Identifiability is basically a model property which describes whether its parameters can be identified from its output for a specified input [3]. Further, the terms structural identifiability [3], practical identifiability [27], and uniqueness are introduced. Structural identifiability focuses on the model function itself and assesses whether identifiability is given or not. In general, it refers to global identifiability. Practical identifiability also considers the sensitivity of the model to the parameters. Practical identifiability requires sensitivity and is related to local identifiability. Uniqueness of the estimated parameter set yields global identifiability.

Sharma and Fathi [38] and Lin and Stefanopoulou [24] used the Fisher information matrix to assess local identifiability. From the Fisher information matrix, the eigenvalues can be used to assess identifiability [10,37], and a singular value decomposition can be carried out. Bizeray et al. linearized a single-particle model to investigate its structural identifiability [3]. This approach has the advantage to be able to assess global identifiability and hence uniqueness but requires a closed formulation of the models output and linearization. Thus, it is not applicable to the commonly used P2D battery model which lacks the closed formulation of its output. Also, it is possible that linearization reduces the model's identifiability. In contrast, the Fisher information matrix could be applied to a P2D model, but computational cost would be enormous for a state-of-the-art desktop PC. For a SPM, the Fisher information matrix was successfully applied by Pozzi et al. to derive optimal experiments for parameterization [31].

Barcellona et al. published a review on parameter identification techniques for lithium-ion battery models [1]. Therein, they were classified in online methods, offline methods, or analytically/numerical calculation methods. Online methods were commonly applied to ECMs for state estimation [1]. Tian et al. estimated parameters of an ECM from constant-current discharge data and assessed local identifiability analyzing the rank of the sensitivity matrix [41]. Pozna et al. showed that even a first-order RC equivalent circuit battery model is not identifiable [30]. For fractional-order models (FOM) of lithium-ion batteries, parameter identifiability was assessed by Guo et al. and Li et al. [13, 
23]. FOM is based on Laplace transformation and provides lumped parameters which have to be estimated.

The review of relevant literature shows the variety of parameter estimation approaches in different scientific communities. The intention of this article is to interconnect those groups and to provide hands-on guidance for parameter estimation of electrochemical models, especially for applied sciences. The advantage of the introduced approach is that it enables estimation of a unique set of model parameters of a P2D model. All existing methods were either global or applicable to the P2D model. In contrast to the published literature of P2D models, it allows assessment of practical identifiability and uniqueness. Thus, it provides the most reliable base for any further physical or electrochemical model-based analysis.

The outline of this article is as follows: In Sect. 2, the model as well as the parameterization strategy is introduced. In Sect. 3, the model is parameterized in three steps. The second step is the common combination of P2D model with C-rate tests. Its practical identifiability is discussed in-depth and a mitigation strategy for its partial unidentifiability is introduced in Sect. 3.3. This article ends with conclusions which provide guidance for the use of electrochemical models in applied sciences and industry.

\section{Mathematical methods}

\subsection{Electrochemical model}

In this article, the common P2D model is applied to simulate C-rate tests. The model was first introduced by Doyle et al. [5, 6]. In the following, the governing equations are briefly reviewed. For a detailed set of equations and boundary conditions, we refer to our previous publication [20]. The model is a set of partial differential and algebraic equations. All spatial derivatives are discretized applying finite volume method.

Intercalation reactions at both electrodes are described by Butler-Volmer kinetics:

$j^{\mathrm{Li}}=a_{\mathrm{s}} i_{0}\left(\exp \left(\alpha \frac{\eta \mathrm{F}}{R T}\right)-\exp \left(-(1-\alpha) \frac{\eta \mathrm{F}}{R T}\right)\right)$

with a concentration-dependent exchange current density $i_{0}$

$i_{0}=k \mathrm{~F} c_{\mathrm{e}}^{\alpha}\left(c_{\max }-c_{\mathrm{s}}\right)^{\alpha} c_{\mathrm{s}}^{1-\alpha}$.

In Eqs. 1 and 2, $j^{\mathrm{Li}}$ is the intercalation current density, $a_{\mathrm{s}}$ the volume-specific active area, $\alpha$ the symmetry coefficient of the reaction, $\eta$ is the electrochemical overpotential, $k$ the reaction rate constant, $c_{\mathrm{e}} \mathrm{Li}^{+}$concentration in electrolyte, $c_{\mathrm{s}}$ the Li concentration in active material, and $c_{\max }$ is the maximal $\mathrm{Li}$ concentration in active material; thus, $c_{\max }-c_{\mathrm{s}}$ are available lattice vacancies for $\mathrm{Li}$ in the active material. Diffusion of lithium in spherical particles is described by Fick's law in a radial coordinate $r$ :

$\frac{\partial c_{\mathrm{s}}}{\partial t}=\frac{1}{r^{2}} \frac{\partial}{\partial r}\left(D_{\mathrm{s}} r^{2} \frac{\partial c_{\mathrm{s}}}{\partial r}\right)$,

wherein $D_{\mathrm{s}}$ is the solid-phase diffusion coefficient. Further, initial values $c_{\mathrm{a}, 0}$ and $c_{\mathrm{c}, 0}$ are introduced for the solid-phase volume elements of anode and cathode at $t=0$ at a cell voltage of $4.2 \mathrm{~V}$, respectively. In the electrolyte, diffusion and migration are considered

$\varepsilon \frac{\partial c_{\mathrm{e}}}{\partial t}=\frac{\partial}{\partial x}\left(D_{\mathrm{e}, \mathrm{eff}} \frac{\partial c_{\mathrm{e}}}{\partial x}\right)+\left(1-t_{\mathrm{p}}\right) \frac{j^{\mathrm{Li}}(x)}{\mathrm{F}}$,

and the liquid-phase potential $\phi_{\mathrm{e}}$ is governed by

$j^{\text {tot }}=-\frac{\partial}{\partial x}\left(\sigma_{\mathrm{e}, \text { eff }} \frac{\partial \phi_{\mathrm{e}}}{\partial x}\right)-2 \frac{R T}{\mathrm{~F}}\left(t_{\mathrm{p}}-1\right) \sigma_{\mathrm{e}, \mathrm{eff}} \frac{\partial \ln c_{\mathrm{e}}}{\partial x}$.

In Eq. $4, \sigma_{\mathrm{e}, \text { eff }}$ is the effective electrolyte conductivity, $\phi_{\mathrm{e}}$ is potential, $x$ is a linear coordinate orthogonal to the plain electrode area, and $t_{\mathrm{p}}$ is the transference number. The effective diffusion coefficient is derived from porosity $\varepsilon$ and tortuosity $\tau$ :

$D_{\mathrm{e}, \mathrm{eff}}=D_{\mathrm{e}, \mathrm{bulk}} \cdot \frac{\varepsilon}{\tau}$.

Further, the model considers electrochemical double layers at both electrodes which was first introduced to this model type by Legrand et al. [21]:

$j^{\mathrm{DL}}=a_{s} C_{\mathrm{DL}} \frac{\partial\left(\phi_{s}-\phi_{e}\right)}{\partial t}$,

with double-layer capacitance $C_{\mathrm{DL}}$ and

$j^{\text {tot }}=j^{\mathrm{Li}}+j^{\mathrm{DL}}$.

At last, the Nernst-Einstein equation is applied to link electrolyte diffusivity $D$, a frequently adjusted model parameter, and electrolyte conductivity $\sigma_{e}$ to reduce the number of independent adjustable parameters:

$\sigma_{\mathrm{e}}=\frac{\mathrm{F}^{2}}{R} \cdot c_{\mathrm{e}} \cdot\left(D^{+}+D^{-}\right)$.

It assumes a linear concentration dependency of $\sigma_{\mathrm{e}}$ and is, thus, a simplification of the model. However, its influence is assumed to be small at low and moderate concentrations.

The open-circuit potential curves are estimated by empirical equations 10 and 11 . The equations are motivated by the work of Smith and Wang [40] but are adjusted to reproduce the characteristics of the given materials more precisely. 


$$
\begin{aligned}
& \begin{aligned}
E_{\mathrm{OCP}, \mathrm{a}}(\tilde{c}) & =k_{1}+k_{2} \tilde{c}+k_{3} \tilde{c}^{0.5}+k_{4} \tilde{c}^{1.5}+k_{5} \exp \left(k_{6}\left(k_{7}-\tilde{c}\right)\right) \\
& +k_{8} \exp \left(k_{9}\left(k_{10}-\tilde{c}\right)\right)+k_{11} \exp \left(k_{12}\left(k_{13}-\tilde{c}\right)\right)+k_{14}\left(k_{15}+\tilde{c}\right)^{-1}
\end{aligned} \\
& E_{\mathrm{OCP}, \mathrm{c}}(\tilde{c})=k_{1} \tilde{c}^{6}+k_{2} \tilde{c}^{5}+k_{3} \tilde{c}^{4}+k_{4} \tilde{c}^{3}+k_{5} \tilde{c}^{2}+k_{6} \tilde{c}+k_{7} \exp \left(k_{8} \tilde{c}^{k_{9}}\right)+k_{10} .
\end{aligned}
$$

The normalized concentration $\tilde{c}$ is given by

$\tilde{c}_{j}=\frac{c_{j}}{\Delta c_{\max , j}}, \forall j \in\{$ an, cath $\}$.

Parameters of Eqs. 10 and 11 are listed in Table 7 in the Online Appendix.

In the end, cell voltage can be derived from the solidphase potential different between the two active material-tocurrent collector interfaces at $x=0$ and $x=L$ :

$U_{\text {cell }}=\phi_{\mathrm{s}}(L)-\phi_{\mathrm{s}}(0)$.

This potential difference includes all potential losses in solid and liquid phases, as well as the OCPs and electrochemical overpotentials of both electrodes.

In total, there are 14 uncertain parameters. For these, the parameter estimation procedure is introduced in the following section. Temperature effects are not considered.

Due to computational efficiency, a single-particle model is used to simulate EIS. Where applicable, the same parameters are used as in the P2D model. For the SEI, there are additional parameters which are taken from Ref. [14] as the SEI is beyond the scope of this study. The model simulated diffusion solely in a single particle per electrode.

The model considers an additional adsorption kinetic at the SEI surface:

$$
\begin{aligned}
r_{\mathrm{Li}, \mathrm{ads}} & =\Theta_{v} a_{\mathrm{s}, \mathrm{ads}} c_{\mathrm{Li}, \mathrm{e}} k_{\mathrm{b}} \exp \left(-\alpha \Delta \phi \frac{\mathrm{F}}{R T}\right) \\
& -\Theta_{\mathrm{Li}} a_{\mathrm{s}, \mathrm{ads}} k_{\mathrm{f}} \exp \left(\alpha \Delta \phi \frac{\mathrm{F}}{R T}\right),
\end{aligned}
$$

wherein a vacant SEI lattice space is $\Theta_{v}$ and a lithium-filled vacancy $\Theta_{\mathrm{Li}}$. Further diffusion and charge transport in the SEI are considered:

Table 2 Steps and parameters of the multi-step PE approach

\begin{tabular}{lll}
\hline PE step & Used experiments & Adjusted parameters \\
\hline 1. Static & OPV & $c_{\mathrm{c}, 0}, c_{\mathrm{a}, 0}, \Delta c_{\mathrm{c}, \max }, \Delta c_{\mathrm{a}, \max }$ \\
2. Quasi-static & C-rate & $D_{\mathrm{s}, \mathrm{c}}, D_{\mathrm{s}, \mathrm{a}}, i_{0, \mathrm{c}}, i_{0, \mathrm{a}}, \tau_{\mathrm{c}}, \tau_{\mathrm{a}}$, \\
& Tests & $\sigma_{\mathrm{s}, \mathrm{c}}, \sigma_{\mathrm{s}, \mathrm{a}}$ \\
3. Dynamic & EIS & $C_{\mathrm{DL}, \mathrm{c}}, C_{\mathrm{DL}, \mathrm{a}}, i_{0, \mathrm{c}}, i_{0, \mathrm{a}}, \sigma_{\mathrm{s}, \mathrm{c}}, \sigma_{\mathrm{s}, \mathrm{a}}$ \\
\hline
\end{tabular}

$$
\begin{aligned}
\frac{\partial c_{\Theta_{\mathrm{Li}}}}{\partial t} & =\frac{\partial}{\partial \xi}\left(D_{\Theta_{\mathrm{Li}}, \mathrm{SEI}} \frac{\partial c_{\Theta_{\mathrm{Li}}}}{\partial \xi}\right), \\
j^{\mathrm{Li}, \text { ads }} & =\frac{\partial}{\partial \xi}\left(\sigma_{\mathrm{SEI}} \frac{\partial \phi_{\mathrm{SEI}}}{\partial \xi}\right) .
\end{aligned}
$$

The linear coordinate in the SEI is denoted $\xi$. However, for details about the single-particle model, it is referred to Ref. [14], as it is only a tool to derive parameters from impedance spectra. It could be substituted by other tools such as equivalent circuit models.

The model was implemented in Matlab R2017b. Multistart fitting was conducted at a high-performance cluster due to its parallelization feasibility. To derive the impedance from simulation data, Matlab built-in fast Fourier transformation was used. For all least-square problems, a Matlab built-in Levenberg-Marquardt algorithm was used.

\subsection{Parameterization procedure}

Based on the literature reviewed above, a three-step parameter estimation procedure is applied in this article. For instance, see Ref. [23] for sequential parameter estimation procedures. The experiments are an OCP measurement for PE step 1, C-rate tests for kinetic parameters in PE step 2, and EIS data for improved identification of kinetic parameters in PE step 3. All experiments are conducted in a threeelectrode setup. The different steps of the applied parameter estimation procedure are summarized in Table 2. This approach uses the higher sensitivity of various experiments to certain parameters to gain more reliable results.

PE step 1 identifies static parameters, like the initial concentration of both electrodes, $c_{\mathrm{a}, 0}$ and $c_{\mathrm{c}, 0}$, and the specific capacity of both active materials, $\Delta c_{\mathrm{a}, \max }$ and $\Delta c_{\mathrm{c}, \max }$ which are not affected by kinetics. PE step 2 is denoted as quasistatic. It is related to kinetic parameters, which are only sensitive at non-zero cell currents. This includes diffusion coefficients of both active materials, respectively, $D_{\mathrm{s}, \mathrm{a}}$ and $D_{\mathrm{s}, \mathrm{c}}$, exchange current densities, $i_{0, \mathrm{a}}$ and $i_{0, \mathrm{c}}$, electric conductivities, $\sigma_{\mathrm{s}, \mathrm{q}}$ and $\sigma_{\mathrm{s}, \mathrm{c}}$, and electrode tortuosities, $\tau_{\mathrm{a}}$ and $\tau_{\mathrm{c}}$, effecting, e.g., the ionic conductivity of the electrolyte. These parameters affect the cell performance at a time scale of seconds to hours. 
The electrochemical cell response at small time scales of seconds and below is considered in PE step 3. It concerns especially reaction kinetics and electric transport. The dynamic cell behavior is analyzed, e.g., via impedance spectroscopy, and the double-layer capacitances, $C_{\mathrm{DL}, \mathrm{a}}$ and $C_{\mathrm{DL}, \mathrm{c}}$, are estimated. Exchange current densities, $i_{0, \mathrm{a}}$ and $i_{0, \mathrm{c}}$, and solid-phase conductivities, $\sigma_{\mathrm{s}, \mathrm{c}}$ and $\sigma_{\mathrm{s}, \mathrm{a}}$, are recalculated, wherein the result of PE step 2 is used as starting value. Solid-phase diffusion coefficients are not recalculated in this step, as they can be estimated precisely in PE step 2. The choice of parameters is based on the literature review in Sect. 1 and especially on the sensitivity analysis conducted in our previous work [20].

In the following, the least-square formulations for all three steps are introduced. As the measured charges and voltages have different magnitudes, the difference between simulation and experiment in the least-square formulations $F_{j}\left(\theta_{j}\right), \forall j \in\{1,2,3\}$ in Eqs. 17 a to $19 \mathrm{~b}$ is normalized vs. the maximum of the respective experimental values. Further, the parameter vectors $\theta_{j}, \forall j \in\{1,2,3\}$ are normalized vs. their starting values for numerical smoothness. For exchange current densities, a logarithmic scale is applied, as the sensitivity of the exchange current density is known to be smaller than the sensitivity of, e.g., diffusion coefficients. This approach is in accordance with the work of, e.g., Jin et al. [17].

As kinetic parameters do not affect the simulated open fullcell voltage curve $E_{\mathrm{OCV}}$ vs. discharged charge $Q$, the static parameters $\theta_{1}^{*}$ are identified in PE step 1 using solely the OCP measurement for the two electrodes $i \in\{$ an, cath $\}$ and $n$ sample points $k$ :

$\theta_{1}^{*}: F\left(\theta_{1}^{*}\right)=\min _{\theta_{1} \in \mathbb{R}_{1}^{N}} F_{1}\left(\theta_{1}\right)$
Both deviations in voltage and charge direction of the simulation from experimental points are considered to ensure accordance for all SOCs. E.g., at intermediate SOCs, the OCV curve is flat and a deviation in charge is large compared to a deviation in capacity. In contrast, at low SOC at a steep OCV curve, deviations in voltage are dominant. Summing up both deviations leads to a high accordance for the entire SOC range. Further, this allows to precisely identify the intercalation steps of the graphite anode.

In PE step 2, the identified static parameters are used. Hence, a subset of kinetic parameters $\theta_{2}^{*}$ is estimated from the C-rate tests with $m$ different $\mathrm{C}$-rates $j$ for the two electrodes, $i \in\{$ an, cath $\}$, and $n$ equidistant sample points $k$ :

$\theta_{2}^{*}: F_{2}\left(\theta_{2}^{*}\right)=\min _{\theta_{2} \in \mathbb{R}_{2}^{N}} F_{2}\left(\theta_{2}\right)$

$F_{2}\left(\theta_{2}\right)=\sum_{i \in\{\text { an }, \text { cath }\}} \sum_{j=1}^{m}\left(\sum_{k=1}^{n}\left(\frac{U_{\text {sim }, i j}\left(C_{k}, \theta_{2}, t\right)-U_{\text {exp }, i j}\left(C_{k}, t\right)}{\max \left(U_{\text {exp }, i}\right)}\right)^{2}\right.$ $\left.+\sum_{k=1}^{n}\left(\frac{C_{\mathrm{sim}, i, j}\left(U_{k}, \theta_{2}\right)-C_{\mathrm{exp}, i, j}\left(U_{k}\right)}{\max \left(C_{\mathrm{exp}, i}\right)}\right)^{2}\right)$.

In PE step 3, EIS is simulated at 50\% SOC. This step is, e.g., in accordance with the approach in Refs. [9] and [8]. The least-square formulation of this step is as follows for the two electrodes $i \in\{$ an, cath $\}$, and $n$ frequencies in the experiment:

$\theta_{3}^{*}: F_{3}\left(\theta_{3}^{*}\right)=\min _{\theta_{3} \in \mathbb{R}_{3}^{N}} F_{3}\left(\theta_{3}\right)$,

$F_{1}\left(\theta_{1}\right)=\sum_{i \in\{\text { an, cath }\}}\left(\sum_{k=1}^{n}\left(\frac{E_{\mathrm{OCV}, \text { sim }, i}\left(Q_{k}, \theta_{1}\right)-E_{\mathrm{OCV}, \exp , i}\left(C_{k}\right)}{\max \left(E_{\mathrm{OCV}, \text { exp }, i}\right)}\right)^{2}+\sum_{k=1}^{n}\left(\frac{Q_{\mathrm{sim}, i}\left(E_{\mathrm{OCV}, k}, \theta_{1}\right)-Q_{\mathrm{exp}, i}\left(E_{\mathrm{OCV}, k}\right)}{\max \left(Q_{\mathrm{exp}, i}\right)}\right)^{2}\right)$.

Table 3 Cell parameters used in the applied model. The parameter set from Ref. [20] is used as reference point for this study

\begin{tabular}{llllll}
\hline Parameter & Symbol & Unit & Anode & Separator & Cathode \\
\hline Layer thickness $^{m}$ & $\delta_{\mathrm{el}}$ & $\mu \mathrm{m}$ & 55.25 & 20.0 & 60.0 \\
Porosity $^{m}$ & $\varepsilon_{\mathrm{e}}$ & - & 0.35 & 0.50 & 0.40 \\
Diffusion coefficient $^{a}$ & $D_{\mathrm{e}}$ & $\mathrm{m}^{2} \mathrm{~s}^{-1}$ & $7.15 \cdot 10^{-9}$ & $7.15 \cdot 10^{-9}$ & $7.15 \cdot 10^{-9}$ \\
Diffusion coefficient $^{a}$ & $D_{\mathrm{s}}$ & $\mathrm{m}^{2} \mathrm{~s}^{-1}$ & $9.35 \cdot 10^{-15}$ & - & $1.10 \cdot 10^{-12}$ \\
Particle size $^{m}$ & $R_{\mathrm{p}}$ & $\mu \mathrm{m}$ & 11.5 & - & 5.5 \\
specific capacity $^{m}$ & $\Delta c_{\mathrm{max}}$ & $\mathrm{mol} \mathrm{L}$ & 24.9 & - & 25.4 \\
Electronic conductivity $^{a}$ & $\sigma_{\mathrm{s}}$ & $\mathrm{mS} \mathrm{m}$ & 20.4 & - & 9.9 \\
Exchange current density $^{-1}$ & $i_{0}$ & $\mathrm{~A} \mathrm{~m}$ & 1.47 & - & 198 \\
Transference number $^{l}$ & $t_{\mathrm{p}}$ & - & 0.24 & 0.24 & 0.24 \\
Charge-transfer coefficient $^{l}$ & $\alpha$ & - & 0.5 & - & 0.5 \\
Double-layer capacitance $^{l}$ & $C_{\mathrm{DL}}$ & $\mathrm{F} \mathrm{m}{ }^{-2}$ & 0.2 & - & 0.2 \\
\hline
\end{tabular}

${ }^{m}$ Measured

${ }^{a}$ Estimated in Ref. [20]

${ }^{l}$ Taken from Ref. [21] 


$$
\begin{aligned}
& F_{3}\left(\theta_{3}\right)=\sum_{i \in\{\mathrm{an}, \mathrm{cath}\}}\left(\sum_{k=1}^{n}\left(\frac{\operatorname{Re}\left(Z_{\text {sim }, i, k}\left(\theta_{3}\right)\right)-\operatorname{Re}\left(Z_{\text {exp }, i, k}\right)}{\max \left(\operatorname{Re}\left(Z_{\text {exp }, i, k}\right)\right)}\right)^{2}\right. \\
& \left.+\sum_{k=1}^{n}\left(\frac{\operatorname{Im}\left(Z_{\text {sim }, i, k}\left(\theta_{3}\right)\right)-\operatorname{Im}\left(Z_{\text {exp }, i, k}\right)}{\max \left(-\operatorname{Im}\left(Z_{\text {exp }, i, k}\right)\right)}\right)^{2}\right)
\end{aligned}
$$

EIS spans a broad frequency range and enables to consider the different time constants of the dynamic processes. This allows to distinguish between parameters related to fast electrochemical reactions and slow diffusion processes. Impedance data, thus, lead to further sensitive parameters like double-layer capacitances of both electrodes. These have a negligible impact in C-rate tests, but a significant impact on the impedance spectra.

\subsection{Electrochemical reference system}

The model is parameterized using experimental data of NMC111 vs. graphite cell. The electrodes are made at a pilot plant-scale production line in the Battery LabFactory Braunschweig. For a detailed description of electrode compositions and production processes, it is referred to our previous publication [20]. Geometric properties and transport process parameters of the electrochemical reference system are listed in Table 3.

All tests were conducted in a commercial three-electrode setup of EL-Cells GmbH, which was placed in a temperature chamber at $25{ }^{\circ} \mathrm{C}$. Test protocols of C-rate tests and OCP measurements are given in Ref. [20]. EIS was conducted at 65 logarithmic-equidistant frequencies ranging from 20 $\mathrm{mHz}$ to $50 \mathrm{kHz}$.

\subsection{Multi-start identifiability test}

In the following, a sample-based identifiability test is introduced. In principle, this test can be applied to any of the three PE steps. Here however, it is solely applied to PE step 2 as this is the main step having the most adjustable parameters. This decision will be justified by the results of Sects. 3.1 and 3.3.

Direct approaches to access identifiability require a closed formulation of the model equation. The P2D model, however, does not satisfy this requirement. A solution to get a closed formulation could be a drastic simplification of the model and linearization. However, the nonlinearity bears a significant amount of information which enhances the identifiability of the model parameters significantly as shown by Lenze et al. [22]. Thus, an indirect, sample-based approach is chosen: multi-start parameter estimation. In this approach, different starting points $\theta_{\mathrm{m}, 0}$, in the domain of model parameters $\Omega_{\mathrm{m}}$, are chosen for the parameter estimation algorithm. The domain $\Omega_{\mathrm{m}, j}$ of parameter $j$ is limited by physically plausible parameter values $x_{\mathrm{lb}, j}$ at the lower boundary (lb) and $x_{\mathrm{ub}, j}$ at the upper boundary (ub) provided by the literature.

The choice of the various starting points can be guided by different approaches. The parameter space could be sampled in an equidistant mesh. This would lead to a large number of sample points and to many unlikely parameter combinations. Random sampling could reduce the number of sample points but would lead to non-deterministic results. Further, a small number of parameter vectors could be chosen by physical insight. This could reduce the number of chosen unlikely parameter combinations, such as that all parameters are at the lower boundary, which would lead to negligible dischargeable capacity. To minimize computational cost, this approach is applied as explained in the following.

Not all parameters show a similar sensitivity towards C-rate curves, so it is focused on the sensitive parameters, as identified in our previous study [20]. Namely this is solidphase diffusion coefficient of both electrodes, electric phase conductivity of both electrodes, and the exchange current densities of both electrodes. Starting values are varied in the following for those parameters.

For each dimension $j$ of the parameter space $\Omega_{\mathrm{m}}$ three values are defined: the boundaries $x_{j, \mathrm{lb}}$ and $x_{j, \mathrm{ub}}$ and the reference starting value $x_{j, \text { ref }}$ listed in Table 3 . From those three values, three potential starting points $x_{j, l, 0}, \forall l \in\{-, 0,+\}$ are derived:

$x_{j,-, 0}=f\left(\log \left(x_{j, \text { ref }, 0}\right), \log \left(x_{j, \mathrm{~b}}\right)\right)$,

$x_{j, 0,0}=x_{j, \text { ref }}$,

$x_{j,+, 0}=f\left(\log \left(x_{j, \mathrm{ref}}\right), \log \left(x_{j, \mathrm{ub}}\right)\right)$.

The applied logarithm gives weight to the fact that literature values for some parameters range over several orders of magnitude, and thus, the starting values should do as well. The sample generator function, Eqs. 20a to 20c, should be adjusted to the individual parameter estimation problem.

\begin{tabular}{|c|c|c|c|c|}
\hline Parameter & $c_{\mathrm{c}, 0}$ in $\mathrm{mol} \mathrm{L}^{-1}$ & $c_{\mathrm{a}, 0}$ in $\mathrm{mol} \mathrm{L}^{-1}$ & $\Delta c_{\mathrm{c}, \max }$ in $\mathrm{mol} \mathrm{L}^{-1}$ & $\Delta c_{\mathrm{a}, \max }$ in $\mathrm{mol} \mathrm{L} \mathrm{L}^{-1}$ \\
\hline Value & 0.025 & 24.87 & 25.41 & 24.88 \\
\hline
\end{tabular}

Table 4 Estimated parameter set from PE step 1 
As three potential starting values for six parameters would lead to a total of 729 different starting points, promising starting points for PE step 2 are chosen by combination of following deterministic rules:

- At least one parameter is set to $x_{j,-, 0}$,

- At least one parameter is set to $x_{j,+, 0}$,

- At least three parameters are set to $x_{j, 0,0}$.

This reduces the number of starting points to 151 . The reference starting point $x_{j, 0}=x_{j, 0,0}, \forall j$ is added as starting point number 152. Further, starting points will be rejected if the discharge capacity at $0.5 \mathrm{C}$ is below $50 \%$ of the experimental value. If a starting point is not rejected, parameter estimation step 2 is conducted as introduced above.

\subsection{Point estimate method}

In Sect. 3.2, a sensitivity analysis is conducted. A nested point estimate method (PEM) is applied. This method is a tool for global sensitivity analysis which is based on deterministic sampling and Taylor series expansion. For details about the method, it is referred to Refs. [35] and [20]. Sensitivity analysis was shown to be feasible to assess accuracy of parameters, e.g., by Refs. [32] and [43].

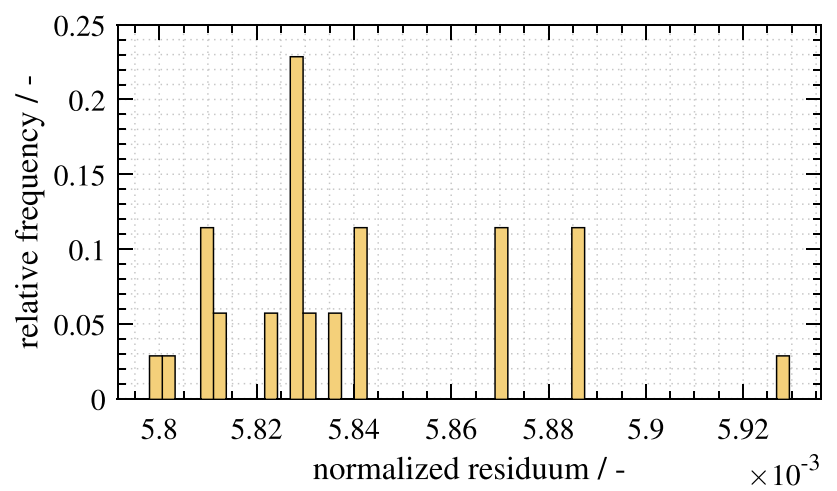

Fig. 1 Histogram of the normalized residuum $F_{2}$ of the entity of parameter estimations which fulfilled the initial requirement of at least $50 \%$ of the experimental capacity at $0.5 \mathrm{C}$

\section{Results and discussion}

In this section, parameter estimation steps 1 to 3 are conducted. For steps 1 and 3, identifiabily is discussed briefly, while for PE, step 2 multi-start fitting is applied. The focus is on identifying a unique parameter set of the P2D model for simulation of quasi-static electrochemical tests, as this was a common combination as shown above.

\subsection{Identifiability of full-cell open-circuit potentials}

The essential part of PE step 1 is to quantify initial capacity and active material losses during the formation at the end of cell production. PE step 1 affects four parameters: $c_{\mathrm{c}, 0}, c_{\mathrm{a}, 0}, \Delta c_{\mathrm{c}, \max }$, and $\Delta c_{\mathrm{a}, \max }$. Only the measurement of halfcell open-circuit potentials is considered. As OCPs are formulated as functions vs. intercalation ratio, respectively, normalized concentration $c_{\mathrm{s}} / \Delta c_{\max }, \Delta c_{\max }$ can be used to stretch the half-cell voltage of one electrode compared to full-cell SOC. The initial concentration $c_{\mathrm{s}, 0}$ of one electrode can be used to shift one electrode vs. full-cell SOC. However, PE step 1 is quite straightforward as the model interpolates the experimental OCP curve with solely an altered base. Basically, PE step 1 is a fine tuning of the altered base.

Results of PE step 1 are listed in Table 4. It is noteworthy that all four parameters describe the technical usable concentration range, analog to full-SOC, not the entire stoichiometric range of $\mathrm{LiC}_{6}$ and $\mathrm{LiNi}_{1 / 3} \mathrm{Mn}_{1 / 3} \mathrm{Co}_{1 / 3}$. For some active materials, the later can be significantly higher than the technical usable range.

To access uniqueness of the estimated parameter set, it is referred to the characteristic shape of both electrode OCP curves, respectively, their nonlinearity. Characteristics of any graphite-based anode's OCP are a wide flat range with discrete step and a steep section at high discharge capacities (low SOCs). In contrast, the NMC cathode's OPC is decreasing with increasing discharge capacity, while the slope is changing as well. For illustration, it is referred to the experimental OCP curves in Fig. 5 in the Online Appendix. The nonlinearity of the OCPs and the availability of half-cell potentials allow identification of $c_{j, 0}$ and $\Delta c_{j, \max }$ for both electrodes. As this approach is based on the characteristics of the OCP curves, it is potentially only valid for the given well-balanced NMC vs. graphite cell. For cells with different OCP characteristics or a

Table 5 Exemplary parameter sets from multi-start parameter estimation

\begin{tabular}{ccccccc}
\hline $\begin{array}{c}\text { parameter } \\
\text { unit }\end{array}$ & $\begin{array}{c}D_{\mathrm{s}, \mathrm{a}} \\
\mathrm{m} \mathrm{s}^{-2}\end{array}$ & $\begin{array}{c}D_{\mathrm{s}, \mathrm{c}} \\
\mathrm{m} \mathrm{s}^{-2}\end{array}$ & $\begin{array}{c}\sigma_{\mathrm{s}, \mathrm{a}} \\
\mathrm{S} \mathrm{m}^{-1}\end{array}$ & $\begin{array}{c}\sigma_{\mathrm{s}, \mathrm{c}} \\
\mathrm{S} \mathrm{m}^{-1}\end{array}$ & $\begin{array}{c}i_{0, \mathrm{a}} \\
\mathrm{A} \mathrm{m}^{-2}\end{array}$ & $\begin{array}{c}i_{0, \mathrm{c}} \\
\mathrm{A} \mathrm{m}^{-2}\end{array}$ \\
\hline parameter set 1 & $1.17 \times 10^{-14}$ & $6.72 \times 10^{-13}$ & 81.3 & 25.0 & 0.38 & 1.36 \\
parameter set 2 & $1.23 \times 10^{-14}$ & $3.47 \times 10^{-13}$ & 0.68 & 0.055 & 0.37 & 2.40 \\
\hline
\end{tabular}

Green highlights a positive change of the parameter between set 1 and set 2, red highlights a negative change. Simulated C-rate tests for both parameter sets are shown in Fig. 6 in the Online Appendix 


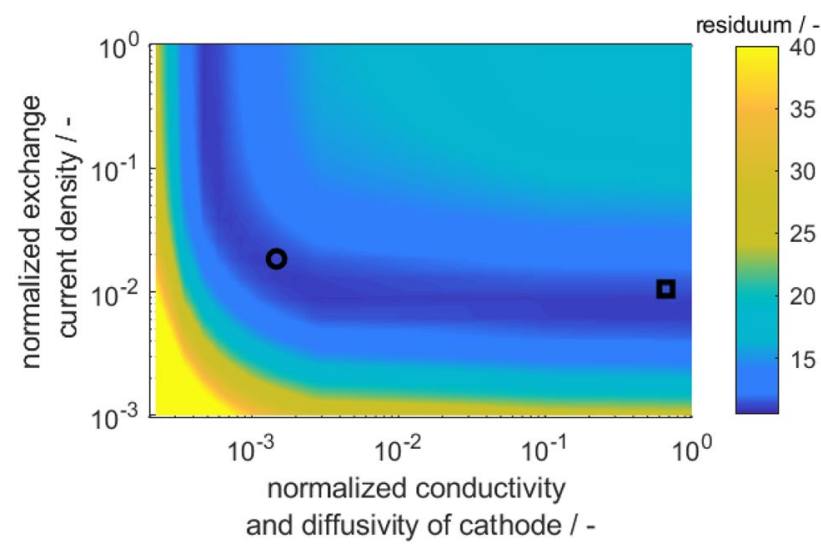

Fig. 2 2D parameter variation to illustrate unidentifiability of the P2D model from C-rate tests with three estimated parameters. The colormap shows the residuum of Eqs. 18a and 18b. The black square marks and the black circle mark parameter sets 1 and 2, respectively

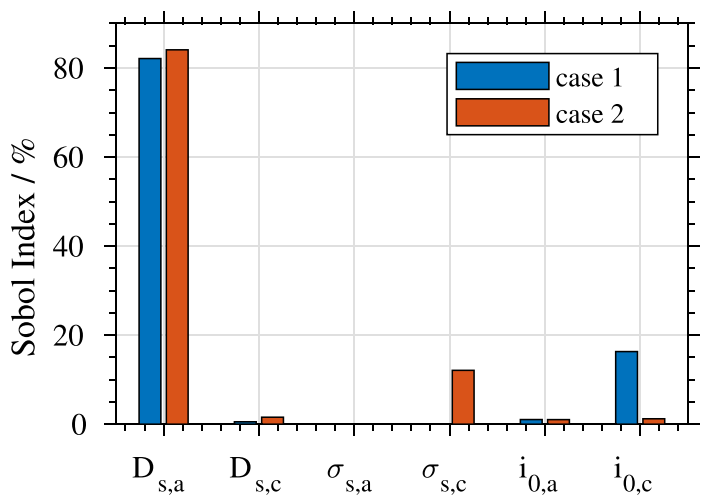

Fig. 3 Sobol indices for both parameter sets from Table 5. Point estimate method was applied for six normally distributed uncertain parameters

different balancing, a different approach can be more suitable. Also, analytic approaches at distinct points may be possible.

\subsection{Practical identifiability from C-rate tests}

Multi-start parameter estimation is conducted for PE step 2 as described in Sect. 2.4.

Out of 152 initial points, 49 have been chosen by the algorithm due to the rejection criterion introduced in Sect. 2.4. Further, results with a normalized residuum below $6.0 \times 10^{-3}$ were chosen for the further analysis. Consequently, the number of parameter vectors is reduced to 35 . Figure 1 shows the normalized residuum of the parameter estimation defined in Eqs. 18a and 18b. There is a narrow band of parameter sets with a residuum between $5.8 \times 10^{-3}$ and $6.0 \times 10^{-3}$. Thus, they only vary about $3.3 \%$ and all are equivalent to an excellent accordance between simulation and experimental C-rate test. Thus, the least-square solver is able to converge for a large number of initial points. In this context, solver convergence implies that any parameter set is found which leads to a minimal residuum of the objective function. In the following, it is assessed whether the solver converges to the same parameter set.

For further discussion of the P2D model's unidentifiability in PE step 2, two parameter sets are handpicked. Both sets lead to an excellent accordance between the experimental discharge curve and the simulation but show significant differences in their parameter values. The two parameter sets are listed in Table 5. The corresponding simulated discharge curves are shown in Fig. 6 in the Online Appendix. Differences between the curves are negligible and below the typical experimental accuracy.

Despite their equal discharge curves, the two different parameter sets vary significantly regarding the cathode properties. The cathode solid-phase diffusivity is decreased between the first and second parameter sets by a factor of two, solid-phase conductivity by a factor of 450 , and the exchange current density is increased by a factor of 70 . At the anode, only the solid-phase conductivity is changed significantly.

Further, a 2D parameter variation is conducted wherein cathode exchange current density is varied (first dimension) and cathode solid-phase conductivity and simultaneously cathode solid-phase diffusivity (second dimension) are varied simultaneously. It is ensured that both parameter sets in Table 5 are a sample of this parameter variation. In Fig. 2, they are highlighted by a circle and a square, respectively. Results of the parameter variation are shown in Fig. 2. It displays $F_{2}\left(\theta_{2}^{*}\right)$ for all samples.

The parameter variation reveals the unidentifiability of the P2D model with C-rate test data, as an infinite number of solutions is visible. Thus, the combined impact of diffusion and conduction in cathode particles is not distinguishable from the impact of the surface reaction. To overcome this limitation, EIS data are used for the model identification in the following section. The different time constants of reaction and transport processes are used for separation of

Table 6 Cathode conductivity and cathode exchange current density estimated from impedance data

\begin{tabular}{llll}
\hline Parameter & Estimated value & Literature value & Refs. \\
\hline$\sigma_{\mathrm{s}, \mathrm{c}}$ in $\mathrm{S} \mathrm{m}^{-1}$ & 0.18 & 0.11 & {$[15]$} \\
$i_{0, \mathrm{c}}$ in $\mathrm{A} \mathrm{m}^{-2}$ & 7 & 1.20 & {$[43]$} \\
\hline
\end{tabular}



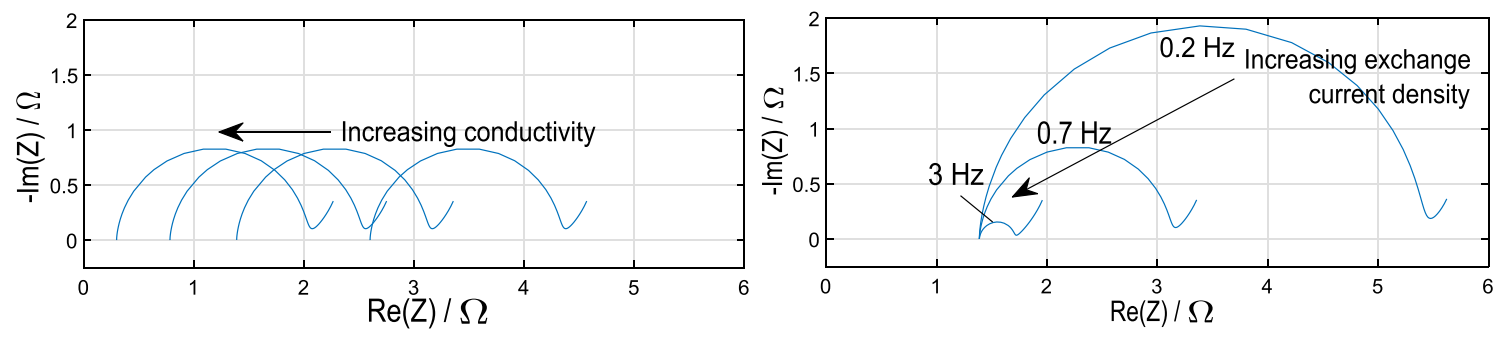

Fig. 4 Impedance spectrum at 50\% SOC of the NMC cathode. (left) Cathode solid-phase conductivity is varied. Characteristic frequency is about $0.7 \mathrm{~Hz}$ for all simulations. (right) Cathode exchange current density is varied. Respective characteristic frequencies are given in the plot

cathode exchange current density and cathode solid-phase conductivity.

To further illustrate the cause of unidentifiability, a sensitivity analysis is carried out for the two different parameter sets, both providing an optimal accordance of simulated and measured C-rate test. Sobol indices are determined for the sensitivity of the residuum applying PEM as introduced in Sect. 2.5. Results are displayed in Fig. 3.

The model is most sensitive to $D_{\mathrm{s}, \mathrm{a}}$, followed by either $\sigma_{\mathrm{s}, \mathrm{c}}$ or $i_{\mathrm{c}, 0}$ in dependence on the case. This reveals the transition between the two arcs of the L-shape of the minimal residuum in Fig. 2. One arc relates potential losses to the surface overpotential of the cathode, while the other arc relates them to ohmic losses in the cathode. Sobol indices of $D_{\mathrm{s}, \mathrm{c}}$ and $i_{\mathrm{a}, 0}$ are marginal, and for $\sigma_{\mathrm{s}, \mathrm{a}}$, they are zero for both cases. Due to that, the significant changes of anodic solid-phase conductivity between the two parameter sets do not affect the discharge curve of the cell, respectively, the residuum $F_{2}$. Further, this leads to practical unidentifiability of the anode conductivity due to non-sensitivity. This further explains the large difference of anode conductivity in Table 5. To enable identifiability of the anode conductivity, a further experiment could be added or the conductivity could be measured ex situ.

Concluding PE step 2, out of the adjusted parameters, the anode solid-phase conductivity remains unidentified as it is insensitive for the investigated C-rates. Further, cathode exchange current density and cathode solid-phase conductivity remain unidentified due to their ambiguous impact on the discharge curve. To overcome this ambiguity, PE step 3 is conducted in the following. The reader is not commended to take PE step 2 as a step-by-step guide for parameter estimation as the optimal parameter estimation procedure may be dependent on applied active materials and electrode design. However, it is emphasized to the different tools, alongside others multi-start parameter estimation and sensitivity analysis, to assess reliability of the estimated parameter set.

\subsection{Identifiability by addition of assessment of impedance spectra}

Electrochemical impedance spectroscopy reveals real and imaginary parts of the battery impedance $Z$ at different frequencies of the sinusoidal current or voltage input signal. At different frequencies, the impedance is governed by different processes and semicircles are related to electrochemical reactions at particle surfaces or in the SEI [15]. Figure 7 in the Online Appendix shows the simulated impedance spectrum of the lithium-ion battery at 50\% SOC for illustration. Commonly, the cathodic impedance contains a single semicircle representing electrochemical reactions and charge of double layers, a low-frequency arc representing slow diffusion processes, and a positive non-zero real part at an imaginary part of zero at high frequencies. This point is related to the conductivity of electrolyte in cathode and separator. The full-cell impedance spectrum contains beside anode and cathode semicircle a further semicircle at high frequencies, which is related to the SEI [15].

Applying the SPM and PE step 3, solid-phase conductivity $\sigma_{\mathrm{s}, \mathrm{c}}$ and exchange current density $i_{0, \mathrm{c}}$ are estimated as listed in Table 6. Simulation and experimental data of the identified cathode are shown in Fig. 8 in the Online Appendix. The linear part of the spectra at high frequencies is an artifact from the lithium reference electrode. Also, a cathode double-layer capacity of $4.18 \mathrm{~F} \mathrm{~m}^{-2}$ was estimated.

Reference values from the literature are also listed in Table 6, which are qualitatively in good accordance to the value determined based on simulations. It should be pointed out that Heins and Schröder used exactly the same electrodes as in this work [15]. Vazquez-Arenas had different electrodes but at least the same active material [43]. The parameters derived from EIS are in good accordance with one of the infinite parameter sets derived from the $\mathrm{C}$-rate test. Thus, this parameter set indicated by the black square is considered as the final parameter set of the P2D model after PE step 3. Cathode diffusivity is taken from PE step 2 from 
the parameter set matching $\sigma_{\mathrm{s}, \mathrm{c}}$ and $i_{0, \mathrm{c}}$ of PE step 3. This approach is based on the results of Beelen et al. [2]. They suggest that for parameterization, tests should be used which are similar to the later application. In this case, a strong focus lies on the P2D model and C-rate tests as the most common combination.

In this study, the objective of PE step 3 is to distinguish between cathode solid-phase conductivity $\sigma_{\mathrm{s}, \mathrm{c}}$ and cathode exchange current density $j_{0, c}$, as the subordinated objective is to parameterize the P2D model for C-rates. Further parameterization of the SPM model and its SEI parameters are beyond the scope of this work. However, for different application a wider parameter study on the impedance data may be beneficial.

To illustrate the feasibility of EIS to do this, the influence of parameter variations of $\sigma_{\mathrm{s}, \mathrm{c}}$ and $j_{0, \mathrm{c}}$ are shown in Fig. 4 for the cathode impedance spectrum.

With increasing solid-phase conductivity, the semicircle moves to lower real parts of impedance, while the size of semicircle stays the same. If the exchange current density is increased, the semicircle, related to cathode intercalation reaction, is decreasing in size, while the abscissa intercept at high frequencies remain the same. Thus, with position and size of the cathode semicircle, the two parameters $\sigma_{\mathrm{s}, \mathrm{c}}$ and $j_{0, \mathrm{c}}$ can be distinguished. It is noteworthy that the abscissa intercept also depends on the electrolyte conductivity. However, this is not an adjustable parameter of the applied model. The electrolyte diffusivity is identified in PE step 2 and the electrolyte conductivity is derived therefrom by the Nernst-Einstein equation.

Further, it is referred to studies on the structural identifiability of a linearized SPM from EIS data of Bizeray et al. They concluded that besides the two solid-phase diffusion coefficients, a lumped charge-transfer resistance could be identified [3]. However, it has to be noticed that their studies were based on full-cell data only. Thus, if half-cell potentials are available, as in our case, separate charge transfer resistances of both electrodes are identifiable.

In conclusion, a three-step parameter estimation procedure was conducted. Out of the 14 adjustable parameters of the P2D model only, the anode solid-phase conductivity remains unidentifiable as it is insensitive at the investigated C-rates. In this case, a reasonable value from literature can be chosen.

\section{Conclusions}

In this article, a P2D model was parameterized in a threestep procedure, and practical identifiability was assessed. Unique parameters of the P2D model were successfully identified through a combination of OCP, C-rate, and EIS data in a three-electrode setup. It was shown that the classical C-rate test in combination with OCP measurements does not provide sufficient information for $\mathrm{P} 2 \mathrm{D}$ model identification. This result is remarkable as the combination of C-rate tests and P2D models is the most common case in literature. Specifically, reaction and conductivity coefficients cannot be distinguished. This may lead to wrong conclusions on limiting processes and losses in the cell. Further, it may mislead cell and electrode optimization to a potentially wrong direction of attention. Further in this study, the anodic solidphase conductivity is unidentifiable due to non-sensitivity in C-rate tests. This is related to the commonly high conductivity of graphite anodes. The conducted study leads to general guidelines for model parameterization concerning identifiability. First, parameterization of an electrochemical model requires a combination of dynamic and constantcurrent measurements. Second, half-cell and full-cell measurements are required. The parameterization procedure and insights in this work can provide guidance for researchers and developers to conduct a proper parameterization of their models. It gives helpful advice and sensitizes the issue of non-uniqueness.

Supplementary Information The online version contains supplementary material available at https://doi.org/10.1007/s10800-021-01579-5.

Acknowledgements This research was funded by the German Federal Ministry for Economic Affairs and Energy (BMWi) through the project "Data-Mining in der Produktion von Lithium-Ionen Batteriezellen (DaLion)" Grant Number 03ET6089. The authors gratefully thank the Battery LabFactory Braunschweig (BLB) for electrode production and the Institute of Environmental and Sustainable Chemistry (IÖNC) for executing of the electrochemical test.

Funding Open Access funding enabled and organized by Projekt DEAL.

\section{Declarations}

Conflict of interest The authors declare that they have no conflict of interest.

Open Access This article is licensed under a Creative Commons Attribution 4.0 International License, which permits use, sharing, adaptation, distribution and reproduction in any medium or format, as long as you give appropriate credit to the original author(s) and the source, provide a link to the Creative Commons licence, and indicate if changes were made. The images or other third party material in this article are included in the article's Creative Commons licence, unless indicated otherwise in a credit line to the material. If material is not included in the article's Creative Commons licence and your intended use is not permitted by statutory regulation or exceeds the permitted use, you will need to obtain permission directly from the copyright holder. To view a copy of this licence, visit http://creativecommons.org/licenses/by/4.0/. 


\section{References}

1. Barcellona S, Piegari L (2017) Lithium ion battery models and parameter identification techniques. Energies. https://doi.org/10. 3390/en10122007

2. Beelen HPGJ, Bergveld HJ, Donkers MC (2018) On experiment design for parameter estimation of equivalent-circuit battery models. In: 2018 IEEE Conference on Control Technology and Applications (CCTA). https://doi.org/10.1109/CCTA.2018.8511529

3. Bizeray AM, Kim JH, Duncan SR, Howey D (2018) Identifiability and parameter estimation of the single particle lithium-ion battery model. IEEE Trans Control Syst Technol. https://doi.org/10.1109/ TCST.2018.2838097

4. Chun H, Han S (2018) Electrochemical model parameter estimation of a lithium-ion battery using a metaheuristic algorithm: cascaded improved harmony search.IFAC-PapersOnLine 51(28):409-413. https://doi.org/10.1016/j.ifacol.2018.11.737

5. Doyle M (1993) Modeling of galvanostatic charge and discharge of thelithium/polymer/insertion cell. J Electrochem Soc 140(6):1526. https://doi.org/10.1149/1.2221597

6. Doyle M, Newman J (1995) The use of mathematical modeling in the design oflithium/polymer battery systems. Electrochim Acta 40(13-14):2191-2196. https://doi.org/10.1016/0013-4686(95) 00162-8

7. Dvorak D, Bauml T, Holzinger A, Popp H (2018) A comprehensive algorithm for estimating lithium-ion battery parameters from measurements. IEEE Trans Sustain Energy 9(2):771-779. https:// doi.org/10.1109/TSTE.2017.2761406

8. Ecker M, Kabitz S, Laresgoiti I, Sauer DU (2015) Parameterization of a physico-chemical model of a lithium-ion battery: II. Model validation. J Electrochem Soc 162(9):A1849-A1857. https://doi.org/10.1149/2.0541509jes

9. Ecker M, Tran TKD, Dechent P, Kabitz S, Warnecke A, Sauer DU (2015) Parameterization of a physico-chemical model of a lithium-ion battery: I. Determination of parameters. J Electrochem Soc 162(9):A1836-A1848. https://doi.org/10.1149/2.0551509jes

10. Forman JC, Moura SJ, Stein JL, Fathy HK (2011) Genetic parameter identification of the Doyle-Fuller-Newman model from experimental cycling of a $\mathrm{LiFePO}_{4}$ battery. In: Proceedings of the 2011 American Control Conferene, p 362-369. https://doi.org/10.1109/ ACC.2011.5991183. http://ieeexplore.ieee.org/document/59911 $83 /$

11. Forman JC, Moura SJ, Stein JL, Fathy HK (2012) Genetic identification and fisher identifiability analysis of the Doyle-FullerNewman model from experimental cycling of a $\mathrm{LiFePO}_{4}$ cell. J. Power Sources 210:263-275. https://doi.org/10.1016/j.jpowsour. 2012.03.009

12. Franco AA (2013) Multiscale modelling and numerical simulation of rechargeable lithium ion batteries: concepts, methods and challenges. RSC Adv 3:13027-13058. https://doi.org/10.1039/c3ra2 $3502 \mathrm{e}$

13. Guo D, Yang G (2018) Parameter identification method for fractional-order model of lithium-ion battery. 2018 IEEE Int. Power Electron. Appl. Conf. Expo. 1(2):1415-1420. https://doi.org/10. 1109/PEAC.2018.8590274

14. Heinrich M, Wolff N, Harting N, Laue V, Röder F, Seitz S, Krewer U (2019) Physico-chemical modeling of a lithium-ion battery: an ageing study with electrochemical impedance spectroscopy. Batter Supercaps 2(6):530-540

15. Heins TP, Schlüter N, Schröder U (2017) Electrode-resolved monitoring of the ageing of large-scale lithium-ion cells by using electrochemical impedance spectroscopy. ChemElectroChem 4(11):2921-2927. https://doi.org/10.1002/celc.201700686

16. Jarraya I, Loukil J, Masmoudi F, Chabchoub MH, Trabelsi H (2018) Modeling and parameters estimation for lithium-ion cells in electric drive vehicle. In: 2018 15th international multi-conference on systems, signals \& devices, p 1128-1132

17. Jin N, Danilov DL, Van den Hof PMJ, Donkers MCF (2018) Parameter estimation of an electrochemistry-based lithium-ion battery model using a two-step procedure and a parameter sensitivity analysis. Int J Energy Res 42(7):2417-2430. https://doi.org/ $10.1002 /$ er.4022

18. Jokar A, Rajabloo B, Désilets M, Lacroix M (2016) Review of simplified pseudo-two-dimensional models of lithium-ion batteries. J Power Sources 327:44-55. https://doi.org/10.1016/j.jpows our.2016.07.036

19. Krewer U, Röder F, Harinath E, Braatz RD, Bedürftig B, Findeisen R (2018) Review-dynamic models of Li-ion batteries for diagnosis and operation: a review and perspective. J Electrochem Soc 165(16):A3656-A3673. https://doi.org/10.1149/2.1061814jes

20. Laue V, Schmidt O, Dreger H, Xie X, Röder F, Schenkendorf R, Kwade A, Krewer U (2019) Model-based uncertainty quantification for the product properties of lithium-ion batteries. Energy Technol. https://doi.org/10.1002/ente.201900201

21. Legrand N, Raël S, Knosp B, Hinaje M, Desprez P, Lapicque F (2014) Including double-layer capacitance in lithium-ion battery models. J. Power Sources 251:370-378. https://doi.org/10.1016/j. jpowsour.2013.11.044

22. Lenze G, Röder F, Bockholt H, Haselrieder W, Kwade A, Krewer U (2017) Simulation-supported analysis of calendering impacts on the performance of lithium-ion-batteries. J Electrochem Soc 164(6):A1223-A1233. https://doi.org/10.1149/2.1141706jes

23. Li X, Pan K, Fan G, Lu R, Zhu C, Rizzoni G, Canova M (2017) A physics-based fractional order model and state of energy estimation for lithium ion batteries. Part II: parameter identification and state of energy estimation for $\mathrm{LiFePO}_{4}$ battery. J Power Sources 367:202-213. https://doi.org/10.1016/j.jpowsour.2017.09.048

24. Lin X, Stefanopoulou AG (2015) Analytic bound on accuracy of battery state and parameter estimation. J Electrochem Soc 162(9):A1879-A1891. https://doi.org/10.1149/2.0791509jes

25. Ma X, Qiu D, Tao Q, Zhu D (2019) State of charge estimation of a lithium ion battery based on adaptive Kalman filter method for an equivalent circuit model. Appl Sci 9:2765

26. Marcicki J, Canova M, Conlisk AT, Rizzoni G (2013) Design and parametrization analysis of a reduced-order electrochemical model of graphite/ $\mathrm{LiFePO}_{4}$ cells for $\mathrm{SOC} / \mathrm{SOH}$ estimation. J Power Sources 237:310-324. https://doi.org/10.1016/j.jpowsour. 2012.12.120

27. Masoudi R, Uchida T, McPhee J (2015) Parameter estimation of an electrochemistry-based lithium-ion battery model. J. Power Sources 291:215-224. https://doi.org/10.1016/j.jpowsour.2015. 04.154

28. Meng J, Luo G, Ricco M, Swierczynski M, Stroe DI, Teodorescu R (2018) Overview of lithium-ion battery modeling methods for state-of-charge estimation in electrical vehicles. Appl Sci 8:659

29. Nejad S, Gladwin DT, Stone DA (2016) A systematic review of lumped-parameter equivalent circuit models for real-time estimation of lithium-ion battery states. J Power Sources 316:183-196. https://doi.org/10.1016/j.jpowsour.2016.03.042

30. Pozna AI, Magyar A, Hangos KM (2017) Model identification and parameter estimation of lithium ion batteries for diagnostic purposes. In: 19th international symposium on power electronics (Ee) 2017-December, p 1-6. https://doi.org/10.1109/PEE.2017. 8171673

31. Pozzi A, Ciaramella G, Volkwein S, Raimondo DM (2018) Optimal design of experiments for a lithium-ion cell: parameters identification of a single particle model with electrolyte dynamics. Ind Eng Chem Res 58:1286-1299. https://doi.org/10.1021/acs.iecr. 8 b04580

32. Ramadesigan V, Chen K, Burns NA, Boovaragavan V, Braatz RD, Subramanian VR (2011) Parameter estimation and capacity 
fade analysis of lithium-ion batteries using reformulated models. J Electrochem Soc 158(9):A1048. https://doi.org/10.1149/1.36099 26

33. Ramadesigan V, Northrop PWC, De S, Santhanagopalan S, Braatz RD, Subramanian VR (2012) Modeling and simulation of lithiumion batteries from a systems engineering perspective. J Electrochem Soc 159(3):R31-R45. https://doi.org/10.1149/2.018203jes

34. Röder F, Laue V, Krewer U (2019) Model based multiscale analysis of film formation in lithium-ion batteries. Batter Supercaps. https://doi.org/10.1002/batt.201800107

35. Schenkendorf R (2014) A general framework for uncertainty propagation based on point estimate methods. In: Proceedings of the 2nd European Conference of the Prognostics and Health Management Society. Fort Worth

36. Schmalstieg J, Sauer DU (2018) Full cell parameterization of a high-power lithium-ion battery for a physico-chemical model: part II. Thermal parameters and validation. J Electrochem Soc 165(16):A3811-A3819. https://doi.org/10.1149/2.0331816jes

37. Schmidt AP, Bitzer M, Imre AW, Guzzella L (2010) Experimentdriven electrochemical modeling and systematic parameterization for a lithium-ion battery cell. J Power Sources 195(15):50715080. https://doi.org/10.1016/j.jpowsour.2010.02.029

38. Sharma A, Fathy HK (2014) Fisher identifiability analysis for a periodically-excited equivalent-circuit lithium-ion battery model. In: Proceedings of the American Control Conference, p 274-280. https://doi.org/10.1109/ACC.2014.6859360

39. Singh P, Chen C, Tan CM, Huang SC (2019) Semi-empirical capacity fading model for soh estimation of Li-ion batteries. Appl Sci 9:3012

\section{Authors and Affiliations}

\section{Vincent Laue ${ }^{1}$. Fridolin Röder ${ }^{1}$ - Ulrike Krewer ${ }^{1,2}$ (D)}

\section{Ulrike Krewer}

ulrike.krewer@kit.edu

1 Institute of Energy and Process Systems Engineering, Technische Universität Braunschweig, Franz-Liszt-Straße 35, 38106 Braunschweig, Germany
40. Smith K, Wang CY (2006) Power and thermal characterization of a lithium-ion battery pack for hybrid-electric vehicles. J. Power Sources 160(1):662-673. https://doi.org/10.1016/j.jpowsour.2006. 01.038

41. Tian N, Wang Y, Chen J, Fang H (2017) On parameter identification of an equivalent circuit model for lithium-ion batteries. In: 1st Annual IEEE Conference on Control Technology and Applications (CCTA) 2017-January, p 187-192. https://doi.org/10.1109/ CCTA.2017.8062461

42. Van CN, Vinh TN (2020) Soc estimation of the lithium-ion battery pack using a sigma point Kalman filter based on a cell's second order dynamic model. Appl Sci 10:1896

43. Vazquez-Arenas J, Gimenez LE, Fowler M, Han T, Chen SK (2014) A rapid estimation and sensitivity analysis of parameters describing the behavior of commercial Li-ion batteries including thermal analysis. Energy Convers Manag 87:472-482

44. Wang Y, Zhao L, Cheng J, Zhou J, Wang S (2020) A state of charge estimation method of lithium-ion battery based on fused open circuit voltage curve. Appl Sci 10:1264

45. Zhang C, Li K, McLoone S, Yang Z (2014) Battery modelling methods for electric vehicles-a review. In: 2014 European Control Conference (ECC), p 2673-2678. https://doi.org/10.1109/ ECC.2014.6862541

Publisher's note Springer Nature remains neutral with regard to jurisdictional claims in published maps and institutional affiliations.

2 Institute for Applied Materials - Electrochemical Technologies, Karlsruhe Institute of Technology, Adenauerring 20b, 76131 Karlsruhe, Germany 